\title{
Diagnostic performance of a novel high-resolution dedicated axillary PET system in the assessment of regional nodal spread of disease in early breast cancer
}

\author{
Jingyi Cheng ${ }^{1,2,3 \#}$, Junjie Li ${ }^{2,4,5 \#}$, Guangyu Liu ${ }^{2,4,5 \#}$, Ruohong Shui ${ }^{2,6}$, Sheng Chen ${ }^{2,4,5}$, Benlong Yang ${ }^{2,4,5}$, \\ Zhimin Shao ${ }^{2,4,5}$
}

${ }^{1}$ Department of Nuclear Medicine, Fudan University Shanghai Cancer Center, Shanghai, China; ${ }^{2}$ Department of Oncology, Shanghai Medical College, Fudan University, Shanghai, China; ${ }^{3}$ Department of Nuclear Medicine, Shanghai Proton and Heavy Ion Center, Fudan University Cancer Hospital, Shanghai, China; ${ }^{4}$ Department of Breast Surgery, Fudan University Shanghai Cancer Center, ${ }^{5}$ Key Laboratory of Breast Cancer in Shanghai, Fudan University Shanghai Cancer Center, Fudan University, Shanghai, China; ${ }^{6}$ Department of Pathology, Fudan University Shanghai Cancer Center, Shanghai, China;

Contributions: (I) Conception and design: Z Shao, J Li; (II) Administrative support: Z Shao; (III) Provision of study materials or patients: J Li, G Liu, S Chen, B Yang, Z Shao; (IV) Collection and assembly of data: J Cheng, J Li; (V) Data analysis and interpretation: J Cheng, J Li, G Liu; (VI) Manuscript writing: All authors; (VII) Final approval of manuscript: All authors.

"These authors contributed equally to this work.

Correspondence to: Zhimin Shao, MD. Department of Breast Surgery, Fudan University Shanghai Cancer Center, 270 Dong-An Road, Shanghai 200032, China. Email: zhimin_shao@yeah.net.

Background: In early breast cancer, a non-invasive method with higher sensitivity and negative predictive value (NPV) is needed to identify and recognize more indolent axillary lymph nodes (ALNs). This study aimed to assess whether a novel high-resolution dedicated ALN positron emission tomography (LymphPET) system could improve sensitivity in detecting early breast cancer (clinical N0-N1 stage).

Methods: A total of 103 patients with clinical stage T1-2N0-1M0 breast cancer were evaluated by ${ }^{18}$ F-fluorodeoxyglucose $\left({ }^{18} \mathrm{~F}-\mathrm{FDG}\right)$ LymphPET. The maximum single-voxel PET uptake value of ALNs (maxLUV) and the tumor-to-background ratio (TBR) for fat (TBR1) and muscle (TBR2) tissue were calculated. Then, 78 patients with cN0 stage breast cancer received sentinel lymph node biopsy alone or combined with axillary lymph node dissection (ALND), and 25 patients with $\mathrm{cN1}$ stage breast cancer underwent fine-needle aspiration.

Results: A total of 99 invasive breast carcinoma cases were included in this study. The diagnostic sensitivity of LymphPET was $88 \%$, specificity was $79 \%$, false-negative rate was $12 \%$, the false-positive rate was $21 \%$, positive predictive value was $75 \%$, NPV was $90 \%$, and accuracy was $83 \%$. The maxLUV was superior to TBR1 and TBR2 in detecting ALNs, with 0.27 being the most optimal cutoff value.

Conclusions: The ${ }^{18}$ F-FDG LymphPET system can be used to identify and recognize more indolent ALNs of breast cancer due to greater sensitivity and a much higher NPV.

Keywords: Lymph node assessment; molecular imaging; new PET technology

Submitted Apr 03, 2021. Accepted for publication Sep 08, 2021.

doi: 10.21037/qims-21-388

View this article at: https://dx.doi.org/10.21037/qims-21-388 


\section{Introduction}

Over the last 2 decades, with improved systemic therapies and molecular tools, breast surgery has moved from surgical axillary lymph node dissection (ALND) to the much less radical surgical sentinel lymph node excision biopsy (SLNB), which is the current reference standard. Currently, there is a global paradigm shift toward de-escalation of axillary lymph node (ALN) surgery, called "less is more". The consensus-based on recent evidence suggests a lack of benefit from overaggressive treatment (1-3). In order to avoid overtreatment, a non-invasive method with higher sensitivity and negative predictive value (NPV) is needed to identify and recognize more indolent ALNs of breast cancer.

${ }^{18} \mathrm{~F}$-fluorodeoxyglucose $\left({ }^{18} \mathrm{~F}\right.$-FDG $)$ whole-body positron emission tomography/computed tomography (WB-PET/ $\mathrm{CT}$ ) has been widely used in assessing the response to neoadjuvant treatment of primary tumors $(4,5)$ and for identifying distant metastases. However, this technique is not ideal in the evaluation of ALN status in early breast cancer. This is mainly because current WB-PET/CT systems typically yield reconstructed images with a spatial resolution of 5-15 $\mathrm{mm}$ depending on the injected dose, imaging time, post-reconstruction filtering, as well as the intrinsic resolution of the scanner. This reduces their utility in detecting small lesions $(<1 \mathrm{~cm})$ and/or those with low tracer uptake (6). As a result, although some authors have found detectability rates as high as $92 \%$ with WB-PET/CT, these were achieved with tumors larger than $2 \mathrm{~cm}$ (7).

Newer imaging techniques offer exciting potential to assess the lower risk and small size of ALNs more sensitively. Dedicated ALN PET (LymphPET) is a new technological development of PEMTECH Co., Ltd. Not only is the spatial resolution of LymphPET much higher than that of WB-PET/CT, but it is also more sensitive in the detection of small, hot, ${ }^{18} \mathrm{~F}-\mathrm{FDG}$-avid lesions, such as subclinical ALNs.

This study aimed to assess whether LymphPET could improve diagnostic performance in early breast carcinoma (clinical N0-N1 stage) and if the NPV would be high enough to recognize more indolent ALNs non-invasively.

\section{Methods}

This retrospective, single-center study included patients whose primary breast cancer was confirmed to be malignant by core needle biopsy. ${ }^{18} \mathrm{~F}$-FDG LymphPET was performed before ALN assessment (ALNA). The LymphPET results were compared with the classical "gold standard" pathological assessment of ALNs, including SLNB alone or in combination with ALND for resectable tumors, and fine-needle aspiration (FNA) and neoadjuvant treatment for unresectable tumors.

\section{Patients}

Patients aged between 18 and 70 years with clinical T12N0-1M0 (cT1-2N0-1M0) breast cancer were enrolled from September to December 2019. Tumor staging was based on the eighth edition of the American Joint Committee on Cancer staging manual. Compared with the pathological $\mathrm{N}$ category $(\mathrm{pN})$, which is based on the microscopic assessment of at least one node, including imaging and diagnostic biopsy, the clinical $\mathrm{N}$ category $(\mathrm{cN})$ is based on physical examination, imaging, FNA or core needle biopsy, and sentinel node biopsy. The cN0 category means no regional lymph metastases (by imaging or clinical examination), while $\mathrm{cN} 1$ means metastases to movable ipsilateral level IIALNs (8). In our study, both the primary and ALN status were evaluated by ultrasound (US). For cN0 patients, ALNs were pathologically assessed using SLNB alone or SLNB and ALND. For cN1, ALNs were pathologically confirmed using FNA.

The exclusion criteria included: uncontrolled diabetes, pregnancy or breastfeeding, had already undergone neoadjuvant treatment, SLNB, or ALND, or had any clinical evidence of distant metastases at the time of enrolment. The study conformed to the Declaration of Helsinki (2013 revision) provisions and was approved by the Institutional Ethics Committee with signed informed consent from every patient before participation in this study.

\section{LymphPET system and examination}

The LymphPET (PEMTECHTM, Shanghai, China) device contains movable double-planar confronted detectors with an axillary view and an adjustable distance between the 2 detector plates from 8 to $37 \mathrm{~cm}$. The size of the sensitive detection area is $208 \times 208 \mathrm{~mm}$ with a useful field of vision (FOV) of $204 \mathrm{~mm} \times 204 \mathrm{~mm}$, with 16 modules in each plate consisting of LYSO crystals with the dimensions $1.2 \mathrm{~mm} \times 1.2 \mathrm{~mm} \times 12 \mathrm{~mm}$, which are coupled to a new double readout module (DRM). The crystal matrices are optically coupled on both ends through thin glass light 

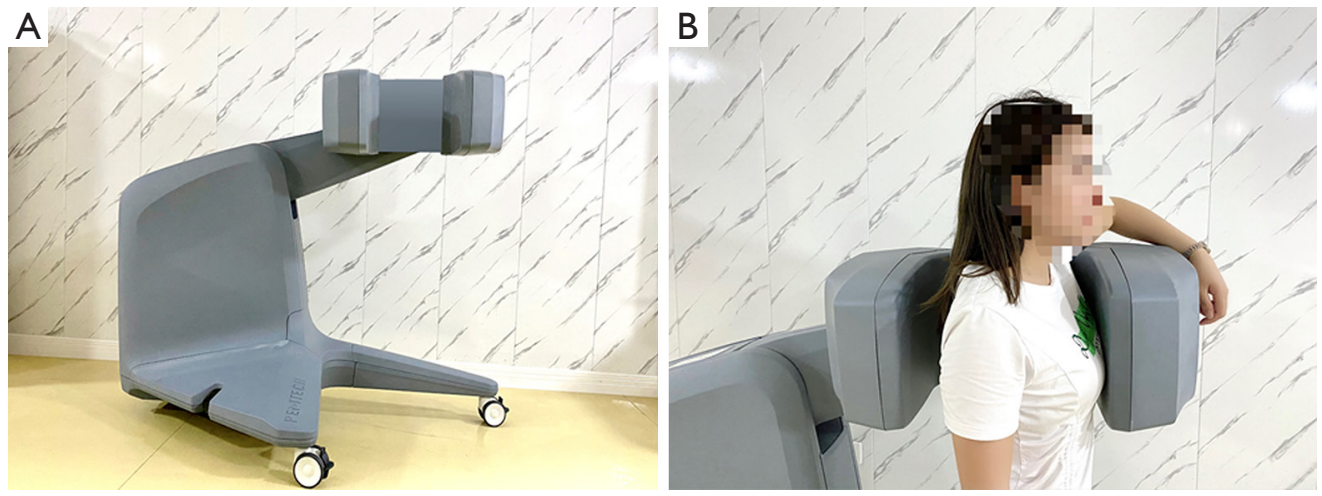

Figure 1 The dedicated axillary lymph node positron emission tomography (LymphPET) system. This image is published with the patient/ participant's consent. (A) The whole device of LymphPET. (B) The acquisition and patient's position.

guides to arrays of $8 \times 8$ individual silicon photomultipliers (SiPMs) using time-of-flight (TOF). Spatial resolution: $<1.4 \mathrm{~mm}$ full width at half maximum (FWHM) at $1 \mathrm{~cm}$ off-center, a uniformity of $10 \%$, energy resolution: $<12 \%$ FWHM at $511 \mathrm{keV}$, timing resolution of $500 \mathrm{ps}$, true coincidence rate peak of $390 \mathrm{kcps}$ at $20 \mathrm{MBq}$, and depthof-interaction (DOI) resolution: $<3 \mathrm{~mm}$. LymphPET is the high resolution because of the following mechanism. Unlike WB-PET/CT, which has a large gantry where the PET ring is mounted, LymphPET is designed to use bi-planar detectors to observe specific regions of interest (ROIs). For example, when it is used to detect the metastatic status of ALNs, the patient's axilla is positioned in the middle of the bi-planar detectors, and the detectors close to the patient lead to a higher sensitivity. Each square-shaped detector plane is designed $20 \mathrm{~cm} \times 20 \mathrm{~cm}$ in size and composed of 16 units of "double-sided front-end readout modules (DRM)" that integrate the LYSO crystal arrays and 2 SiPM arrays frontend electronics in a compact detector module. In order to deliver better sensitivity, spatial resolution, and timing resolution, a SiPM is used instead of a traditional photomultiplier tube (PMT) (9). By measuring light signals to compare the ratio between light outputs detected at one side and both sides, the double-sided module design has an advantage in providing continuous DOI information with better DOI resolution. DOI information will improve time resolution and enhance spatial resolution, even at the center of the FOV (10).

Patients were fasted at least $6 \mathrm{~h}$ before receiving a standardized injection of $4 \mathrm{mCi}{ }^{18} \mathrm{~F}-\mathrm{FDG}$ (injection in the contralateral arm to the breast lesion). Blood glucose levels were required to be lower than $10 \mathrm{mmol} / \mathrm{L}$. After a resting period of 60 minutes to allow tracer distribution,
LymphPET was performed, and the acquisition duration was 3 minutes each region, and bilateral axillary regions were detected one by one. The patient sat down on a fixed chair comfortably with their upper arm raised and supported by a dedicated bracket (Figure 1). During acquisition, the patient was asked to maintain stability. After the acquisition, the images were checked immediately. If there were anatomical motion, the acquisition would be performed again. Images were reconstructed with a 3D standard maximum likelihood expectation maximization (MLEM 3D) algorithm with a voxel size of $0.5 \times 0.5 \times 0.5 \mathrm{~mm}^{3}(11)$. The matrix was obtained over the computation of every solid voxel angle related to the total volume surface. We found 15 iterations which represented the ideal accuracy to reconstruction time.

\section{LymphPET image analysis}

In order to postulate a precise method of quantification adapted to this geometry, the concept of the LymphPET uptake value (LUV), as a surrogate for the standardized uptake value (SUV), has been used for this special biplanar symmetry. The LUV, like the SUV, characterizes the activity intensity in the image, but unlike the SUV, the quantification of LUV does not suffer the same data corrections such as attenuation or scattered photons $(12,13)$. Although the validity of the LUV as a quantification metric has not yet been demonstrated due to its novelty, a similar concept is generally accepted (14-16). A single large study published in the AJR demonstrated a statistically significant correlation between the PEM uptake value (PUV) and the SUV in the breast (15).

Image evaluation and quantification of the maximum single-voxel LUV (maxLUV) were performed using 
commercial Medical Image Merge (version 6.5.4; MI M Software Inc., OH, USA), which is the professional image processing software certified by the United States Food and Drug Administration. Two experienced nuclear medicine physicians with 10 years of experience in PET/CT, who were blinded to study-related information apart from the laterality of breast cancer, independently analyzed the images and marked the ROIs manually.

ALNs were considered positive under the following three conditions: (I) the positive focus was located in the axillary region but not in the skin, muscle, or bone; (II) ${ }^{18}$ F-FDG uptake was higher than the reference background (fat tissue); and (III) the physiological lymphatic uptake was excluded, such as symmetrically bilateral positive focus, like 2 funicular ropes. For quantitative analysis, the minor diameter of the lymph node (LN) was measured, and an elliptic-shaped ROI was drawn manually. ${ }^{18} \mathrm{~F}$-FDG uptake into this ROI was calculated as the ALN maxLUV. The highest maxLUV was selected as the study value in case multiple LNs were detected. Additionally, 3 separate ROIs measuring $1 \mathrm{~cm}$ in diameter (fat background) were located at the axillary adipose tissue, and the mean value of these areas was defined as maxLUV ${ }_{\text {fat }}$. Moreover, $31-\mathrm{cm}$ diameters ROIs were located at the biceps brachii, and ectopectoralis muscles (muscle background), and the mean value was noted as maxLUV $V_{\text {muscle }}$. The tumor-to-background ratio (TBR) was calculated as the coefficient between the positive value of maxLUV and the corresponding background value: $\mathrm{TBR} 1=\operatorname{maxLUV} / \operatorname{maxLUV} \mathrm{fat}_{\mathrm{fa}}$; TBR2 $=\operatorname{maxLUV} / \max L U V_{\text {muscle }}$. Thus, three parameters were calculated for each patient: maxLUV, TBR1, and TBR2.

\section{Surgery and pathological evaluation}

For cN0 patients, ALNs were pathologically assessed using SLNB alone or SLNB and ALND. For cN1 patients, ALNs were pathologically confirmed using FNA. If FNA was negative, SLNB was performed. If FNA was positive, neoadjuvant treatment was performed. For patients with no more than 2 positive SLNs, the decision to perform further axillary dissection depends on the operative type (breast-conserving therapy or mastectomy) and individual pathological characteristics. All LymphPET scans were performed before FNA.

In SLNB, nodes were identified by injection of blue dye (methylthioninium chloride, Jiangsu Jumpcan Pharmaceutical Co., Ltd.) and/or by intraoperative guidance of the gamma detector probe (neo2000, Neoprobe
Corporation) using a radiocolloid ${ }^{99 \mathrm{~m}}$ technetium sulfur colloid, CIS US Inc. Bedford, MA, USA). In our study, SLNs were defined as any blue-stained node, any node with a blue-stained lymphatic channel directly leading to it, any node with radioactive counts $10 \%$ or more of the most radioactive node, or any pathologically palpable nodes. Internal mammary SLNB would not be performed even if the nodes were detected using lymphoscintigraphy.

Touch imprint cytology was routinely performed on every SLN that was harvested. SLNs were cut along the long axis at a $2.0-$ to $3.0-\mathrm{mm}$ interval intraoperatively, and each cut surface was touched, at least 3 times, onto a clean glass slide and stained with hematoxylin and eosin. Staining of seriallysectioned slices was performed in $100-\mu \mathrm{m}$ intervals for every node. Slides were sent for cytopathological examination immediately after preparation. Moreover, additional slices were formalin-fixed and paraffin-embedded for further evaluation. According to the pathological results, ALNs were classified as macro-metastasis $(>2.0 \mathrm{~mm})$, micro-metastasis $(0.2-2.0 \mathrm{~mm}$ ), and isolated tumor cells (ITCs, $<0.2 \mathrm{~mm}$ ) according to the tumor-node-metastasis staging system.

It should be noted that both ITCs and micro-metastases $(<2 \mathrm{~mm})$ were considered negative in the final statistical analysis.

\section{Statistical analysis}

Data were presented as a range with means and standard deviation. Receiver operating characteristic (ROC) curves and the areas under the ROC curves (AUCs) were employed to evaluate the predictive value of ${ }^{18} \mathrm{~F}$-FDG LymphPET, by determining the optimal cutoff for the 3 recorded parameters. Logistic regression analysis was used to determine independent factors predictive of ALN metastases. Multiple linear regression analysis was used to confirm whether maxLUV correlated with different clinical factors. For categorical variables, differences were calculated using the Chi-squared test or Fisher's exact test where applicable. Interclass correlation coefficients (ICCs) were used to evaluate the consistency and reproducibility of the inter-observer agreement of the parameters. An ICC value of $>0.75$ indicated good reliability. Statistical analysis was performed using the SPSS 20.0 software (IBM Inc., Chicago, IL, USA), and twosided $\mathrm{P}$ values were reported with an alpha of $0.05(\mathrm{P}<0.05)$.

\section{Results}

Between September 2019 and December 2019, 103 females 
Table 1 Patient characteristics $(n=99)$

\begin{tabular}{|c|c|c|}
\hline Characteristics & No. & $\%$ \\
\hline Age (years), range/mean & $19-67 / 47.41$ & \\
\hline Weight $(\mathrm{kg})$, range/mean & $43-77 / 58.96$ & \\
\hline Breast tumor size $(\mathrm{mm})$, range/mean & $7-48 / 23.03$ & \\
\hline \multicolumn{3}{|l|}{ Tumor grade } \\
\hline II & 40 & 57.97 \\
\hline III & 29 & 42.03 \\
\hline \multicolumn{3}{|l|}{ T category } \\
\hline $\mathrm{T} 1$ & 35 & 36.45 \\
\hline $\mathrm{T} 2$ & 61 & 63.55 \\
\hline Local resection & 3 & \\
\hline \multicolumn{3}{|l|}{$\mathrm{N}$ category } \\
\hline NO & 74 & 74.75 \\
\hline N1 & 25 & 25.25 \\
\hline \multicolumn{3}{|l|}{ Subtype } \\
\hline Luminal & 66 & 66.67 \\
\hline HER2 & 10 & 10.10 \\
\hline TNBC & 23 & 23.23 \\
\hline \multicolumn{3}{|l|}{ Treatment } \\
\hline Mastectomy & 74 & 74.75 \\
\hline NAT & 25 & 25.25 \\
\hline
\end{tabular}

TNBC, triple-negative breast cancer; NAT, neoadjuvant treatment.

with breast cancer (age range 19-67 years, mean 47.41) were registered. Four cases were excluded because the primary lesions were confirmed to be TIS (tumor in situ, carcinoma in situ, or stage 0 cancer). Finally, 99 cases with invasive carcinoma were included, and the clinical and anatomopathological characteristics of these patients are shown in Table 1. Of these, 41 (41\%) were ALN metastatic cases, and 16 cases were confirmed by SLNB alone or SLNB with ALND, and 25 by FNA (Figure 2).

\section{Diagnostic performance of LymphPET}

The diagnostic sensitivity (88\%), specificity (79\%), falsenegative rate $(12 \%)$, false-positive rate $(21 \%)$, positive predictive value (PPV, 75\%), NPV (90\%), and accuracy $(83 \%)$ of LymphPET are summarized in Figure 3. All 5 false-negative cases were of a luminal subtype with nuclear grade II. Two of them were negative, and 3 displayed enlarged nodes, but no metastasis, in the axillary US. Of these 5 patients, 3 to 5 SLNs were biopsied, and we found 1 macro-metastasis in 3 patients each, 2 macro-metastases in 1 patient, and 1 patient was confirmed by FNA. Then, 4 patients with a maxLUV of "0" underwent ALND with $0 / 16,1 / 16,3 / 16$, and 3/19 in finally ALND surgery, and 1 patient with maxLUV of 0.16 underwent NAC. Twelve false positives (FPs) were identified, including 7 luminal subtypes and 5 triple-negative breast cancer (TNBC) subtypes, with 4 nuclear grade IIs and 8 nuclear grade IIIs. In 2 of 12 patients, axillary US was negative, whereas the remaining 10 patients displayed enlarged LNs measuring up to $20 \mathrm{~mm} \times 30 \mathrm{~mm}$. Three patients with SLNB were identified as micro-metastases. They were luminal subtypes with Ki67 were $5 \%, 10 \%$, and $30 \%$, respectively. The axillary US displayed that 2 of them were negative, and 1 patient had a small LN of $7 \mathrm{~mm} \times 5 \mathrm{~mm}$ (diagnosis was substantial LN in axillary).

\section{Comparison between maxLUV, TBR1, and TBR2}

The mean values of the 3 parameters (maxLUV, TBR1, and TBR2) calculated by both physicians were used for further analysis due to the excellent agreement (ICCs $>0.85$, $\mathrm{P}<0.05)$. A total of 48 patients had positive LymphPET scans in ALNs, with a maxLUV range from 0.16 to 5.05 $(1.13 \pm 1.11)$. The maxLUV fat background ranged from 0.02 to $0.15(0.07 \pm 0.02)$ and the maxLUV $V_{\text {muscle }}$ background ranged from 0.13 to $0.33(0.23 \pm 0.05)$. The TBR1 ranged from 2.66 to $55.75(14.11 \pm 11.88)$, and the TBR2 ranged from 0.94 to $16.93(4.42 \pm 3.65)$. Strong correlations were found between maxLUV and TBR1 $(r=0.81, \mathrm{P}<0.05)$, and maxLUV and TBR2 $(r=0.97, \mathrm{P}<0.05)$. We identified 48 positive nodes, including 36 true-positive (TP) nodes and 12 FP nodes (Table 2). There were statistically significant differences in maxLUV, TBR1, and TBR2 in the TP and FP groups but not in terms of size. These results indicate that maxLUV, TBR1, and TBR2, but not size, could be used to distinguish TP from FP LNs.

ROC curves of the 3 selected parameters for predicting nodal metastasis are shown in Figure 4, and the results of univariate and multivariate analysis are presented in Table 3. The AUC values for maxLUV, TBR1, and TBR2 were 0.895 (95\% CI, 0.828-0.962), 0.885 (95\% CI, 0.815-0.954), and 0.884 (95\% CI, 0.814-0.954), respectively. The diagnostic yield of TBR1 and TBR2 was inferior to that of maxLUV. Therefore, maxLUV was used in the subsequent analysis. 


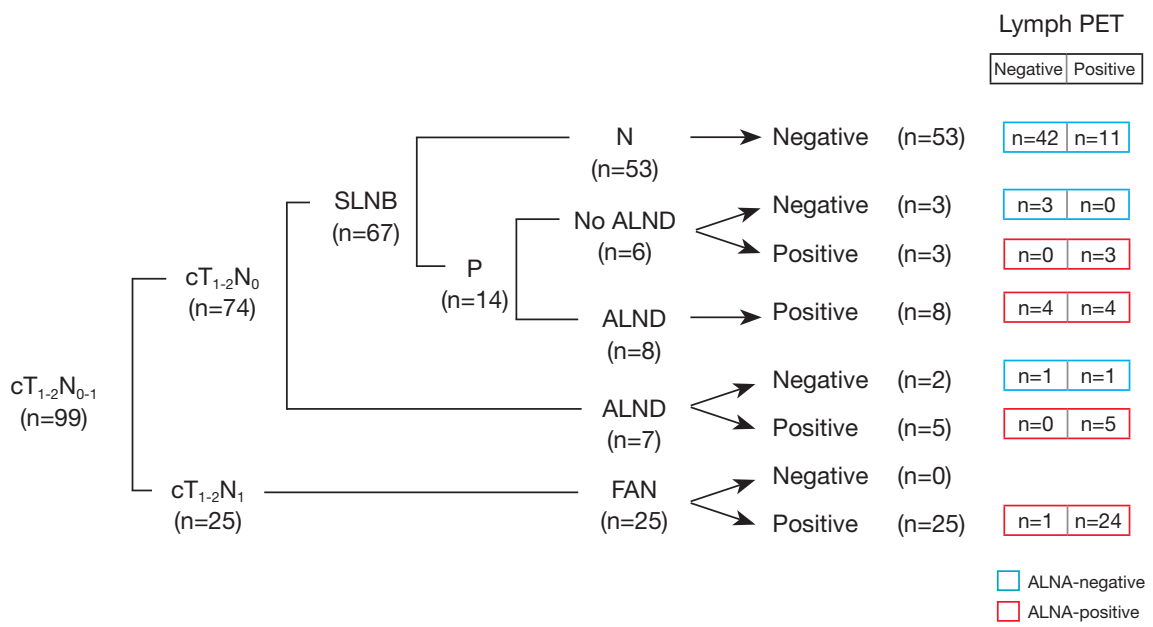

Figure 2 Consort diagram showing the results of ALNA and dedicated axillary positron emission tomography (LymphPET) scanning. ALNA, axillary lymph node assessment; SLNB, sentinel lymph node biopsy; ALND, axillary lymph node dissection.

\begin{tabular}{|c|c|c|c|c|}
\hline \multicolumn{5}{|c|}{ ALNA } \\
\hline \multirow{4}{*}{ 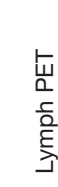 } & & + & - & \multirow{4}{*}{$\begin{array}{l}48 \\
51 \\
99\end{array}$} \\
\hline & & $\mathrm{TP}=36$ & $E P=12$ & \\
\hline & \multirow[t]{2}{*}{-} & $\mathrm{FN}=5$ & $\mathrm{TN}=46$ & \\
\hline & & 41 & 58 & \\
\hline \multicolumn{5}{|c|}{ Sensitivity $=88 \%$} \\
\hline \multicolumn{5}{|c|}{ Specificity $=79 \%$} \\
\hline \multicolumn{5}{|c|}{ False-negative rate $=12 \%$} \\
\hline \multicolumn{5}{|c|}{ False-positive rate $=21 \%$} \\
\hline \multicolumn{5}{|c|}{ Positive predictive value $(\mathrm{PPV})=75 \%$} \\
\hline \multicolumn{5}{|c|}{ Negative predictive value (NPV) $=90 \%$} \\
\hline \multicolumn{5}{|c|}{ Accuracy $=83 \%$} \\
\hline
\end{tabular}

Figure 3 Estimates of diagnostic accuracy. ALNA, axillary lymph node assessment; TP, true positive; FP, false positive; TN, true negative; FN, false negative.

According to the ROC curve and the most optimal Youden's index, when the maxLUV cutoff value was set at 0.27 , the most optimal sensitivity and specificity were $88 \%$ and $79 \%$, respectively. On the other hand, we noted that if the maxLUV cutoff value was set at 0.69 , the specificity was $98 \%$, but the sensitivity was only $51 \%$.

\section{Correlation between maxLUV and other parameters}

In the multivariate analysis, primary tumor size, nuclear grade, ER-positive, HER2 high expression, and the proliferation marker Ki67 were not independent predictive factors of $\mathrm{LN}$ metastasis $(\mathrm{P}>0.05$ for all). Using logistic regression, we found that the maxLUV of LNs was affected by the size of the primary tumor and by Ki67 expression, indicating that the larger the tumor or, the higher the Ki67 level, the greater the chance that LymphPET can correctly detect the absence of nodal disease. However, it should be noted that for positive tests, the obtained results were inconclusive due to a $21 \% \mathrm{FP}$ rate. Typical cases are shown in Figures 5,6.

\section{Discussion}

There is a global paradigm shift toward de-escalation of axillary management based on recent evidence suggesting a lack of benefit from overaggressive treatment (17). Hence, it is important to distinguish between patients who are clinically node positive and patients who are clinically node negative, but who have positive SLNs. Presently, SLNB is the standard initial workup in breast cancer patients who are clinically node negative, with about a $9.8 \%$ false-negative rate (18). A non-invasive molecular tool that can achieve the same false-negative rate will validate the noninferiority with SLNB in future axillary management.

Different companies and projects have been working on this type of open, dedicated PET system (19-21). We have even been testing another prototype with similar geometry (22) but with very different characteristics, both in terms of detectors (simple versus double reading modules), crystals (continuous versus pixelated), mechanics (manual versus automatic), TOF capability (absent versus present), and the software (external versus ad hoc) (23-25). Finally, 
Table 2 Differences in parameters between the TP and FP groups $(\mathrm{n}=48)$

\begin{tabular}{lccc}
\hline Parameter & TP $(n=36)$ & FP $(n=12)$ & $t$ \\
\hline LN size $(\mathrm{mm})$, range & $4-25$ & $4-15.5$ & -1.965 \\
LN size $(\mathrm{mm})$, mean & $10.49 \pm 5.35$ & $7.35 \pm 3.44$ & $0.39>0.05$ \\
maxLUV, range & $0.16-4.9$ & $0.2-0.7$ & -2.778 \\
maxLUV, mean & $1.34 \pm 1.13$ & $0.46 \pm 0.15$ & -2.182 \\
TBR1, range & $2.67-78.75$ & $4-13$ & $0.001<0.05$ \\
TBR1, mean & $17.13 \pm 15.42$ & $7.67 \pm 3.23$ & $0.02<0.05$ \\
TBR2, range & $0.94-16.93$ & $1.25-5.20$ & -2.432 \\
TBR2, mean & $5.13 \pm 3.98$ & $2.40 \pm 1.02$ & $0.001<0.05$ \\
\hline
\end{tabular}

LN size $=$ axillary lymph node size measured by LymphPET. TP, true positive; FP, false positive; TBR, tumor-to-background ratio .

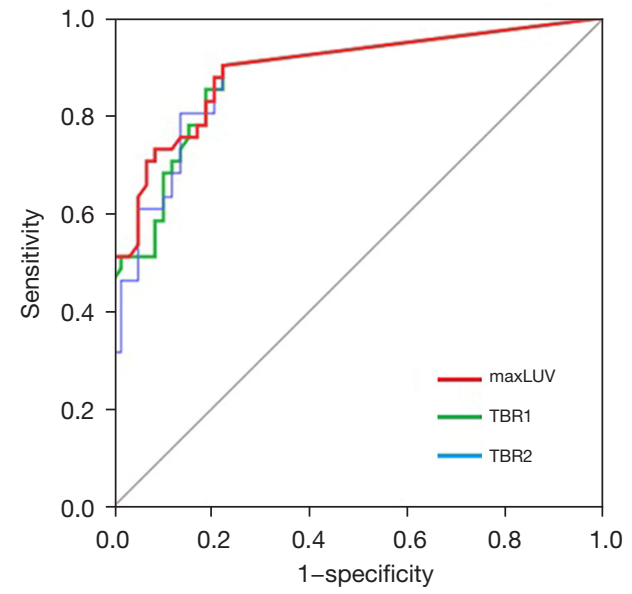

Figure 4 ROC curves of the 3 selected parameters for predicting nodal metastasis. The AUC values for maxLUV, TBR1, and TBR2 were $0.895,0.885$, and 0.884 , respectively. ROC, receiver operating characteristic; AUC, areas under the ROC curve. due to its technical specifications, versatility, and simplicity of use, and the highest resolution and quantification capacity, the system we have selected to go through clinical validation and future studies in patients is the LymphPET $\left(\mathrm{PEMTECH}^{\mathrm{TM}}\right)$.

Current non-invasive diagnostic modalities of ALNs include US, mammography (MMG), magnetic resonance imaging (MRI), and WB-PET/CT. While US remains one of the key tools, it is limited to being subjective (26). A systematic review that analyzed US use showed significant variation between institutions, with overall sensitivity ranging between $26-76 \%$ and a specificity between 88-98\% (27). A more recent meta-analysis involving 21 studies found that US assessment of abnormal nodes allowed a median sensitivity of $64 \%$ and a specificity of $82 \%$ (28). MMG is more suitable for examining breast disease but is not considered reliable for ALN evaluation because part of the axillary area may not be visualized by routine MMG (29).

Table 3 Univariate and multivariate analysis of the 3 parameters $(n=99)$

\begin{tabular}{|c|c|c|c|c|c|c|}
\hline Parameter & No $(n=58)$ & $N 1(n=41)$ & F value & $P$ value & OR $(95 \% \mathrm{Cl})$ & $P$ value \\
\hline TBR1 & $1.72 \pm 3.55$ & $17.27 \pm 21.90$ & 28.29 & $<0.01$ & $1.03(0.80-1.32)$ & 0.81 \\
\hline TBR2 & $0.54 \pm 1.11$ & $4.99 \pm 5.37$ & 37.72 & $<0.01$ & $0.27(0.05-1.43)$ & 0.12 \\
\hline
\end{tabular}



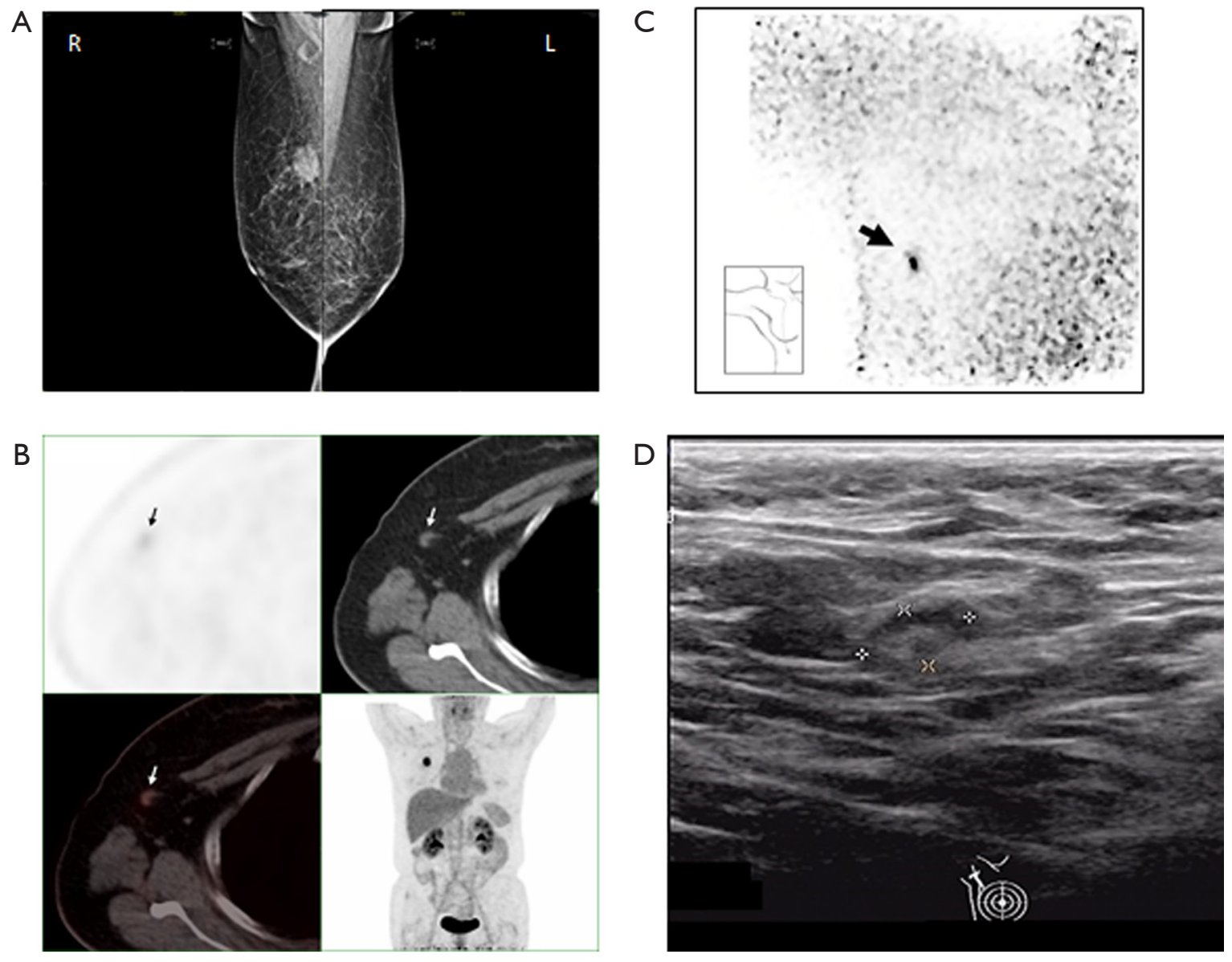

Figure 5 Typical true positive case: 57 year old, right breast cancer (TNBC) without treatment, ER(-), PR(-), HER2(0), Ki67 =40\%. (A) Mammography before surgery: right breast showing a clear mass without ipsilateral axillary positive lymph node evident. (B) Whole body PET/CT. A small lymph node in the right axilla (arrow head) $9 \mathrm{~mm} \times 4 \mathrm{~mm}$ in size and a slight FDG uptake with SUVmax=1.8 (<2.5). (C) LymphPET image. Right axillary lymph node (arrow head) with LUVmax $=1.2(>0.27)$ and $7 \mathrm{~mm} \times 4$ mm size. Pathological diagnosis: right ALN metastasis. Surgery: ALND 3/22(+) with 3 positive lymph nodes. (D) US image. Right axillary penetration and low echo, $8 \mathrm{~mm} \times 5 \mathrm{~mm}$, regular shape, smooth edges, the lymphatic hilum structure is visible, and the cortex is thickened. Diagnosis: substantial lymph node in the right axilla. TNBC, triple-negative breast cancer; US, ultrasound.

MRI is mostly used to assess newly diagnosed breast cancer and examine the response to neoadjuvant treatment, but is also limited by an insufficient imaging field of the axillary region (30). As for WB-PET/CT, several studies showed unacceptably low sensitivity and NPV for cN0 detection. Veronesi et al. (31) compared the value of ${ }^{18} \mathrm{~F}-\mathrm{FDG}$ WB-PET/CT and SLNB in identifying occult axillary metastases. A total of 236 patients with T1-3 primary breast carcinomas and $\mathrm{cN} 0$ were enrolled, and neither patients with ductal carcinoma in situ nor those who had received neoadjuvant chemotherapy were candidates. Results showed that ${ }^{18} \mathrm{~F}$-FDG WB-PET/CT was negative in 193 cases, including 128 true-negative cases and 65 false-negative cases of SLNB. These results suggested that ${ }^{18} \mathrm{~F}-\mathrm{FDG}$ WB-PET/ CT could not detect occult axillary metastases in one-third of cases. Pritchard et al. (32) analyzed 325 operable cT1T3Nx breast cancer cases, and the ALNs were assessed by SLNB and/or ALND. Using ALNA as the gold standard, sensitivity for WB-PET/CT was $23.7 \%$, specificity was 99.6 , PPV was $95.8 \%$, and NPV was $75.4 \%$. These results agreed with other studies, which showed that WB-PET/CT had low sensitivity and NPV in predicting the ALN status $(33,34)$. Therefore, the NCCN guidelines recommend the use of ${ }^{18}$ F-FDG WB-PET/CT in staging breast cancer, starting 
A

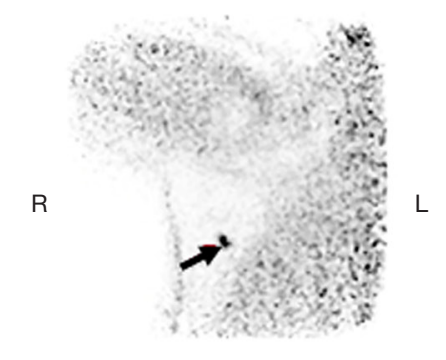

B

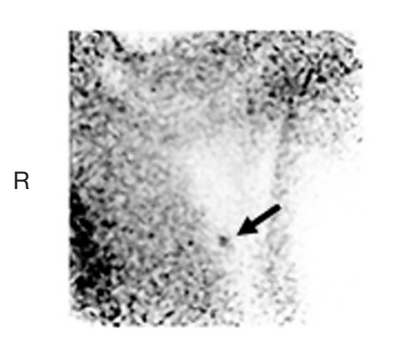

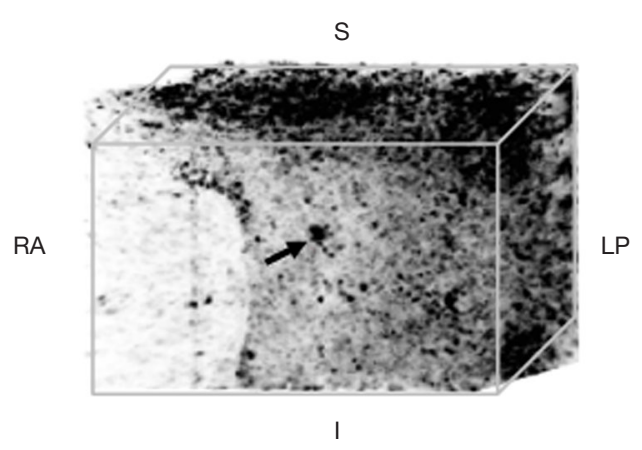

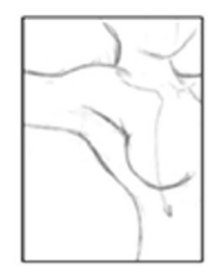

$S$

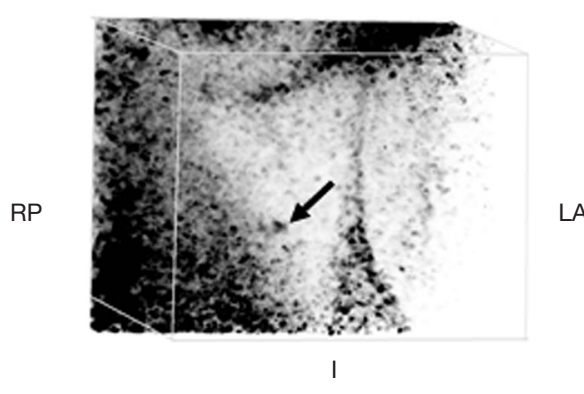

LA

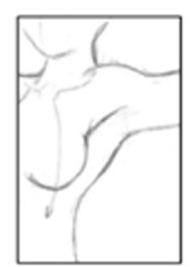

Figure 6 Examples of true positives and false positives with LymphPET. (A) True positive. A 45-year-old woman with right invasive breast cancer (cT1N0M0). LymphPET images demonstrated a $7 \mathrm{~mm} \times 5 \mathrm{~mm}$ axillary node with focused FDG uptake, and the maxLUV was 1.2 (arrow), which was considered to be a metastasis. The patient underwent SLNB which revealed 2 macro-metastases (2/5), and ALND showed no other metastasis (0/20). Single projection (left) and MIP (right) are shown. (B) False positive. A 65-year-old woman with left invasive breast cancer (cT1N0M0). LymphPET showed a $5 \mathrm{~mm} \times 6 \mathrm{~mm}$ node (arrow) with maxLUV $=0.33$. SLNB showed no lymph node metastasis $(0 / 3)$ and the hot node was proven to be a false positive. FDG, fluorodeoxyglucose; maxLUV, maximum single-voxel standard uptake value of the axillary lymph node; SLNB, sentinel lymph node biopsy; ALND, axillary lymph node dissection.

from stage IIIA (T3, N1, and M0) (35).

In the present study, LymphPET significantly increased the diagnostic sensitivity to $88 \%$, thereby reducing the false-negative rate (12\%) and enhancing NPV (90\%). Of all 99 cases, 41 were pathologically confirmed to be macro-metastasis. LymphPET was accurate in diagnosing 36 cases while leading to misdiagnosis in 5 cases, and the NPV (90\%) was much higher than that of WB-PET/CT. Further analysis revealed that these 5 cases of FNs were all of the luminal subtypes with nuclear grade II, suggesting that lower proliferation and metabolism leads to reduced FDG uptake. Additionally, ITCs $(<0.2 \mathrm{~mm})$ and micrometastases $(0.2-2 \mathrm{~mm})$ were defined as pathologically negative because they are too difficult to be detected by any imaging technique, and several recent studies have shown that micro-metastases do not influence overall survival (36-38).

Another aim of this study was to identify the most suitable indicator for detecting ALNs using LymphPET. Therefore, in addition to a new concept LUV, another two indicators (TBR1 and TBR2) were used. Our results demonstrated that while the 3 parameters were highly correlated, maxLUV showed the highest AUC, revealing that it was still the most reliable indicator in LymphPET. When the cutoff value of maxLUV $\geq 0.27$ recommended by Youden's index was confirmed, the sensitivity of diagnosis was $88 \%$, and the NPV reached a maximum of $90 \%$, which is about the same level of NPV as intraoperative cytology $(88.1 \%)(16)$. When the cutoff value of maxLUV $\geq 0.69$ was chosen, a maximum specificity (98\%) was reached. Consequently, the cutoff value of maxLUV should be chosen depending on the clinical context: if a maximum 
NPV is desired, 0.27 is more suitable, whereas a high specificity for confirmation of LN metastasis can be better achieved using 0.69 .

The detection of micro-metastases in LNs depends on better pathological examination techniques for SLNB (immunohistochemical staining and multistep sectioning). Because the size of micro-metastases was no more than 2 $\mathrm{mm}$, the most sophisticated imaging modality will never provide the same information as SLNB. On the other hand, the LymphPET system only involves the PET system without the CT system, and the assessment of the size of LNs based on anatomy was limited. Success in detecting macro-metastases results from the high metabolic rate of the tumor, indicated by Ki67 expression, but not from the large size of the tumor, which is an intrinsic characteristic (39). Actually, using logistic regression analysis, we observed that both the size of the primary lesion (not the size of LNs) as well as Ki67 levels influenced maxLUV, whereby the size of the primary tumor, as well as proliferative activity indicated by Ki67 expression, positively correlated with ALN metastasis. These findings are consistent with previous studies (40) and indicate that LymphPET produces a higher NPV in patients with larger primary lesions, higher Ki67 levels, HER2 subtypes, or TNBC.

The limitation of our study is the absence of WB PET imaging to compare the same patient. A future, prospective study (NCT04072653, SOAPET trial) is ongoing to apply LymphPET to ALN management.

\section{Conclusions}

The ${ }^{18}$ F-FDG LymphPET system could be used to identify and recognize more indolent ALNs of breast cancer due to much higher sensitivity and a lower false-negative rate. The newer imaging techniques raise the possibility that in the future, such techniques may obviate the need for surgical SLNB in selected patient populations.

\section{Acknowledgments}

Funding: None.

\section{Footnote}

Conflicts of Interest: All authors have completed the ICMJE uniform disclosure form (available at https://dx.doi. org/10.21037/qims-21-388). The authors have no conflicts of interest to declare.
Ethical Statement: The authors are accountable for all aspects of the work in ensuring that questions related to the accuracy or integrity of any part of the work are appropriately investigated and resolved. The study was conducted in accordance with the Declaration of Helsinki (as revised in 2013). The study was approved by the Institutional Ethics Committee and signed informed consent was obtained from every patient prior to participation in this study.

Open Access Statement: This is an Open Access article distributed in accordance with the Creative Commons Attribution-NonCommercial-NoDerivs 4.0 International License (CC BY-NC-ND 4.0), which permits the noncommercial replication and distribution of the article with the strict proviso that no changes or edits are made and the original work is properly cited (including links to both the formal publication through the relevant DOI and the license). See: https://creativecommons.org/licenses/by-nc-nd/4.0/.

\section{References}

1. Esserman L, Gallant E, Alvarado M. Less Is More: The Evolving Surgical Approach to Breast Cancer. Am Soc Clin Oncol Educ Book 2016;35:e5-e10.

2. Burstein HJ, Curigliano G, Loibl S, Dubsky P, Gnant M, Poortmans P, Colleoni M, Denkert C, Piccart-Gebhart M, Regan M, Senn HJ, Winer EP, Thurlimann B; Members of the St. Gallen International Consensus Panel on the Primary Therapy of Early Breast Cancer 2019. Estimating the benefits of therapy for early-stage breast cancer: the St. Gallen International Consensus Guidelines for the primary therapy of early breast cancer 2019. Ann Oncol 2019;30:1541-57.

3. Giuliano AE, Hunt KK, Ballman KV, Beitsch PD, Whitworth PW, Blumencranz PW, Leitch AM, Saha S, McCall LM, Morrow M. Axillary dissection vs no axillary dissection in women with invasive breast cancer and sentinel node metastasis: a randomized clinical trial. JAMA 2011;305:569-75.

4. Wu S, Wang Y, Li J, Zhang N, Mo M, Klimberg S, Kaklamani V, Cochet A, Shao Z, Cheng J, Liu G. SubtypeGuided 18 F-FDG PET/CT in Tailoring Axillary Surgery Among Patients with Node-Positive Breast Cancer Treated with Neoadjuvant Chemotherapy: A Feasibility Study. Oncologist 2020;25:e626-33.

5. Cheng J, Wang Y, Mo M, Bao X, Zhang Y, Liu G, Zhang J, Geng D. 18F-fluorodeoxyglucose (FDG) PET/CT after 
two cycles of neoadjuvant therapy may predict response in HER2-negative, but not in HER2-positive breast cancer. Oncotarget 2015;6:29388-95.

6. Koolen BB, Aukema TS, González Martínez AJ, Vogel WV, Caballero Ontanaya L, Vrancken Peeters MJ, Vroonland CJ, Rutgers EJ, Benlloch Baviera JM, Valdés Olmos RA. First clinical experience with a dedicated PET for hanging breast molecular imaging. Q J Nucl Med Mol Imaging 2013;57:92-100.

7. Zhang J, Shi X, Xiao Y, Ma C, Cao G, Liu Y, Li Y. Early SUVmax is the best predictor of axillary lymph node metastasis in stage III breast cancers. Quant Imaging Med Surg 2021;11:1680-91.

8. Giuliano AE, Edge SB, Hortobagyi GN. Eighth Edition of the AJCC Cancer Staging Manual: Breast Cancer. Ann Surg Oncol 2018;25:1783-5.

9. Wagatsuma K, Sakata M, Ishibashi K, Hirayama A, Kawakami H, Miwa K, Suzuki Y, Ishii K. Direct comparison of brain 18FFDG images acquired by SiPMbased and PMT-based PET/CT: phantom and clinical studies. EJNMMI Phys 2020;7:70.

10. Li M, Abbaszadeh S. Depth-of-interaction study of a dualreadout detector based on TOFPET2 application-specific integrated circuit. Phys Med Biol 2019;64:175008.

11. Shepp LA, Vardi Y. Maximum likelihood reconstruction for emission tomography. IEEE Trans Med Imaging 1982;1:113-22.

12. Rahim S, Mawlawi O, Fox P, Taylor S, Millican R, Swanston NM, Brown JE, Rohren EM. Correlation of PUV and SUV in the extremities while using PEM as a high-resolution positron emission scanner. Skeletal Radiol 2014;43:453-8.

13. Shkumat NA, Springer A, Walker CM, Rohren EM, Yang WT, Adrada BE, Arribas E, Carkaci S, Chuang $\mathrm{HH}$, Santiago L, Mawlawi OR. Investigating the limit of detectability of a positron emission mammography device: a phantom study. Med Phys 2011;38:5176-85.

14. MacDonald L, Edwards J, Lewellen T, Haseley D, Rogers J, Kinahan P. Clinical imaging characteristics of the positron emission mammography camera: PEM Flex Solo II. J Nucl Med 2009;50:1666-75.

15. Wang CL, MacDonald LR, Rogers JV, Aravkin A, Haseley DR, Beatty JD. Positron emission mammography: correlation of estrogen receptor, progesterone receptor, and human epidermal growth factor receptor 2 status and 18F-FDG. AJR Am J Roentgenol 2011;197:W247-55.

16. Eo JS, Chun IK, Paeng JC, Kang KW, Lee SM, Han W, Noh DY, Chung JK, Lee DS. Imaging sensitivity of dedicated positron emission mammography in relation to tumor size. Breast 2012;21:66-71.

17. Yan M, Abdi MA, Falkson C. Axillary Management in Breast Cancer Patients: A Comprehensive Review of the Key Trials. Clin Breast Cancer 2018;18:e1251-9.

18. Krag DN, Anderson SJ, Julian TB, Brown AM, Harlow SP, Ashikaga T, Weaver DL, Miller BJ, Jalovec LM, Frazier TG, Noyes RD, Robidoux A, Scarth HM, Mammolito DM, McCready DR, Mamounas EP, Costantino JP, Wolmark N; National Surgical Adjuvant Breast and Bowel Project. Technical outcomes of sentinel-lymph-node resection and conventional axillary-lymph-node dissection in patients with clinically node-negative breast cancer: results from the NSABP B-32 randomised phase III trial. Lancet Oncol 2007;8:881-8.

19. Brem RF, Rapelyea JA, Zisman G, Mohtashemi K, Raub J, Teal CB, Majewski S, Welch BL. Occult breast cancer: scintimammography with high-resolution breast-specific gamma camera in women at high risk for breast cancer. Radiology 2005;237:274-80.

20. Raylman RR, Majewski S, Weisenberger AG, Popov V, Wojcik R, Kross B, Schreiman JS, Bishop HA. Positron emission mammography-guided breast biopsy. J Nucl Med 2001;42:960-6.

21. Weinberg I, Majewski S, Weisenberger A, Markowitz A, Aloj L, Majewski L, Danforth D, Mulshine J, Cowan K, Zujewski J, Chow C, Jones E, Chang V, Berg W, Frank J. Preliminary results for positron emission mammography: real-time functional breast imaging in a conventional mammography gantry. Eur J Nucl Med 1996;23:804-6.

22. Moliner L, Zhang C, Alamo J, Morera C, JiménezSerrano S, Cuallado J, Benlloch J M. Biplanar breast PET: preliminary evaluation. In 15th International Workshop on Breast Imaging (IWBI2020) (Vol. 11513, p. 115131R). International Society for Optics and Photonics. Available online: https://spie.org/Publications/Proceedings/ Paper/10.1117/12.2564346

23. Surti S, Karp JS. Design considerations for a limited angle, dedicated breast, TOF PET scanner. Phys Med Biol 2008;53:2911-21.

24. Lee E, Werner ME, Karp JS, Surti S. Design Optimization of a TOF, Breast PET Scanner. IEEE Trans Nucl Sci 2013;60:1645-52.

25. Krishnamoorthy S, LeGeyt B, Werner ME, Kaul M, Newcomer FM, Karp JS, Surti S. Design and performance of a high spatial resolution, time-of-flight PET detector. IEEE Trans Nucl Sci 2014;61:1092-8.

26. Lee B, Lim AK, Krell J, Satchithananda K, Coombes RC, 
Lewis JS, Stebbing J. The efficacy of axillary ultrasound in the detection of nodal metastasis in breast cancer. AJR Am J Roentgenol 2013;200:W314-20.

27. Alvarez S, Añorbe E, Alcorta P, López F, Alonso I, Cortés J. Role of sonography in the diagnosis of axillary lymph node metastases in breast cancer: a systematic review. AJR Am J Roentgenol 2006;186:1342-8.

28. Houssami N, Ciatto S, Turner RM, Cody HS 3rd, Macaskill P. Preoperative ultrasound-guided needle biopsy of axillary nodes in invasive breast cancer: meta-analysis of its accuracy and utility in staging the axilla. Ann Surg 2011;254:243-51.

29. Shetty MK, Carpenter WS. Sonographic evaluation of isolated abnormal axillary lymph nodes identified on mammograms. J Ultrasound Med 2004;23:63-71.

30. van Nijnatten TJA, Ploumen EH, Schipper RJ, Goorts B, Andriessen EH, Vanwetswinkel S, Schavemaker M, Nelemans P, de Vries B, Beets-Tan RGH, Smidt ML, Lobbes MBI. Routine use of standard breast MRI compared to axillary ultrasound for differentiating between no, limited and advanced axillary nodal disease in newly diagnosed breast cancer patients. Eur J Radiol 2016;85:2288-94.

31. Veronesi U, De Cicco C, Galimberti VE, Fernandez JR, Rotmensz N, Viale G, Spano G, Luini A, Intra M, Veronesi P, Berrettini A, Paganelli G. A comparative study on the value of FDG-PET and sentinel node biopsy to identify occult axillary metastases. Ann Oncol 2007;18:473-8.

32. Pritchard KI, Julian JA, Holloway CM, McCready D, Gulenchyn KY, George R, Hodgson N, Lovrics P, Perera F, Elavathil L, O'Malley FP, Down N, Bodurtha A, Shelley W, Levine MN. Prospective study of $2-\left[{ }^{88} \mathrm{~F}\right]$ fluorodeoxyglucose positron emission tomography in the assessment of regional nodal spread of disease in patients with breast cancer: an Ontario clinical oncology group study. J Clin Oncol 2012;30:1274-9.

33. Gary A Ulaner. PET/CT for Patients With Breast Cancer:
Where Is the Clinical Impact? AJR Am J Roentgenol 2019;2:254-65.

34. Yararbas U, Avci NC, Yeniay L, Argon AM. The value of 18F-FDG PET/CT imaging in breast cancer staging. Bosn J Basic Med Sci 2018;18:72-9.

35. Gradishar WJ, Anderson BO, Abraham J, Aft R, Agnese D, Allison KH, et al. Breast Cancer, Version 3.2020, NCCN Clinical Practice Guidelines in Oncology. J Natl Compr Canc Netw 2020;18:452-78.

36. Donker M, van Tienhoven G, Straver ME, Meijnen P, van de Velde CJ, Mansel RE, et al. Radiotherapy or surgery of the axilla after a positive sentinel node in breast cancer (EORTC 10981-22023 AMAROS): a randomised, multicentre, open-label, phase 3 non-inferiority trial. Lancet Oncol 2014;15:1303-10.

37. Giuliano AE, Hunt KK, Ballman KV, Beitsch PD, Whitworth PW, Blumencranz PW, Leitch AM, Saha S, McCall LM, Morrow M. Axillary dissection vs no axillary dissection in women with invasive breast cancer and sentinel node metastasis: a randomized clinical trial. JAMA 2011;305:569-75.

38. Giuliano AE, Ballman KV, McCall L, Beitsch PD, Brennan MB, Kelemen PR, Ollila DW, Hansen NM, Whitworth PW, Blumencranz PW, Leitch AM, Saha S, Hunt KK, Morrow M. Effect of Axillary Dissection vs No Axillary Dissection on 10-Year Overall Survival Among Women With Invasive Breast Cancer and Sentinel Node Metastasis: The ACOSOG Z0011 (Alliance) Randomized Clinical Trial. JAMA 2017;318:918-26.

39. Kong EJ, Chun KA, Cho IH, Lee SJ. (18)F-FDG PET/ CT with Contrast Enhancement for Evaluation of Axillary Lymph Node Involvement in T1 Breast Cancer. Nucl Med Mol Imaging 2010;44:170-6.

40. Kanyılmaz G, Yavuz BB, Aktan M, Karaağaç M, Uyar M, Findık S. Prognostic Importance of Ki-67 in Breast Cancer and Its Relationship with Other Prognostic Factors. Eur J Breast Health 2019;15:256-61.
Cite this article as: Cheng J, Li J, Liu G, Shui R, Chen S, Yang B, Shao Z. Diagnostic performance of a novel highresolution dedicated axillary PET system in the assessment of regional nodal spread of disease in early breast cancer. Quant Imaging Med Surg 2022;12(2):1109-1120. doi: 10.21037/qims21-388 